https://helda.helsinki.fi

\title{
The Feasibility of Angiosome-Targeted Endovascular Treatment in Patients with Critical Limb Ischaemia and Foot Ulcer
}

\section{Spillerova, Kristyna}

2016

Spillerova , K , Söderström , M , Albäck , A \& Venermo , M 2016 , ' The Feasibility of Angiosome-Targeted Endovascular Treatment in Patients with Critical Limb Ischaemia and Foot Ulcer ' , Annals of Vascular Surgery, vol. 30 , pp. 270-276 . https://doi.org/10.1016/j.avsg.2015.07.020

http://hdl.handle.net/10138/223829

https://doi.org/10.1016/j.avsg.2015.07.020

publishedVersion

Downloaded from Helda, University of Helsinki institutional repository.

This is an electronic reprint of the original article.

This reprint may differ from the original in pagination and typographic detail.

Please cite the original version. 


\title{
The Feasibility of Angiosome-Targeted Endovascular Treatment in Patients with Critical Limb Ischemia and Foot Ulcer
}

\author{
Kristýna Špillerová, Maria Sörderström, Anders Albäck, and Maarit Venermo, Helsinki, \\ Finland
}

Background: To determine wound location according to the angiosome concept and to investigate the feasibility and success of angiosome-based revascularization in below-the-knee (BTK) arteries.

Methods: This was a retrospective study of 161 patients $(67.5 \pm 25.5$ years, $66.5 \%$ diabetics $)$ with critical limb ischemia and a foot ulcer, stage Rutherford 5-6, who underwent percutaneous transluminal angioplasty on BTK arteries in 2012. We evaluated feasibility of angiosome-targeted revascularization and the number of angiosomes affected by a wound in each patient. Patients were divided into 3 groups depending on how many BTK vessels were suitable for revascularization. The feasibility was analyzed in each group and in relation to number of affected angiosomes. Results: The wound(s) interfered with one angiosome in only $39(24.0 \%)$ cases. Direct flow into affected angiosome was successfully achieved in $98(60.9 \%)$ cases. If ulceration was limited in one angiosome, the targeted revascularisation was possible in 27 cases $(69.2 \%)$, if ulceration was extended over 2 angiosomes it was possible in 65 cases (86.7\%), if 3 angiosomes were affected it was possible in 36 cases (85.7\%), when 4 angiosomes were affected the rate dropped to $25.0 \%$ and ulceration extended over 5 angiosomes had no possibility of revascularization.

Conclusions: In critical limb ischemia, the tissue lesion affects several angiosomes in majority of the cases. In thus far published literature, there is existence of more approaches of angiosome-targeted revascularization when more than one angiosome is clinically involved and therefore consensus needs to be achieved for the accurate definition.

\section{INTRODUCTION}

The most severe stage of critical lower limb ischemia (CLI) is tissue lesion, and patients who developed

Funding: The study was funded by a research grant from Finska Läkarsällskapet.

Conflict of Interest: None.

Department of Vascular Surgery, Faculty of Medicine, University of Helsinki and Helsinki University Hospital, Helsinki, Finland.

Correspondence to: Kristýna Špillerová, MD, Department of Vascular Surgery, Helsinki University Central Hospital, P.O. Box 440, FI-00029 HUS, Helsinki, Finland; E-mail: kspillerova@gmail.com

Ann Vasc Surg 2016; 30: 270-276

http://dx.doi.org/10.1016/j.avsg.2015.07.020

(C) 2016 Elsevier Inc. All rights reserved.

Manuscript received: May 14, 2015; manuscript accepted: July 3, 2015; published online: November 11, 2015. such stage have a severe risk of major amputation. Percutaneous transluminal angioplasty (PTA) is increasingly being used as the first-line revascularization procedure in CLI. Despite the increasing endovascular interventions in below-the-knee (BTK) arteries, nonhealing ulcers remain common and very often require additional ulcer surgery to prevent amputation.

Multiple studies about wound healing have been published over the last decade in vascular literature, supporting the primary idea that, without pulsatile flow into the correct foot region, the wound will fail to heal. In 1987, Ian Taylor et al. ${ }^{1}$ presented the angiosome principle in their landmark anatomy study by dividing the body into 3-dimensional anatomic blocks of tissue supplied by a specific artery, the arteriosome. 
A
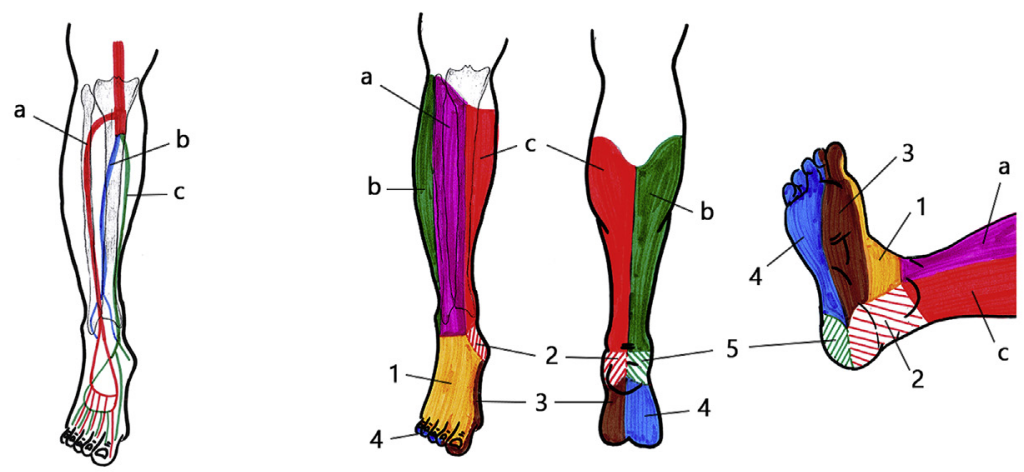

B
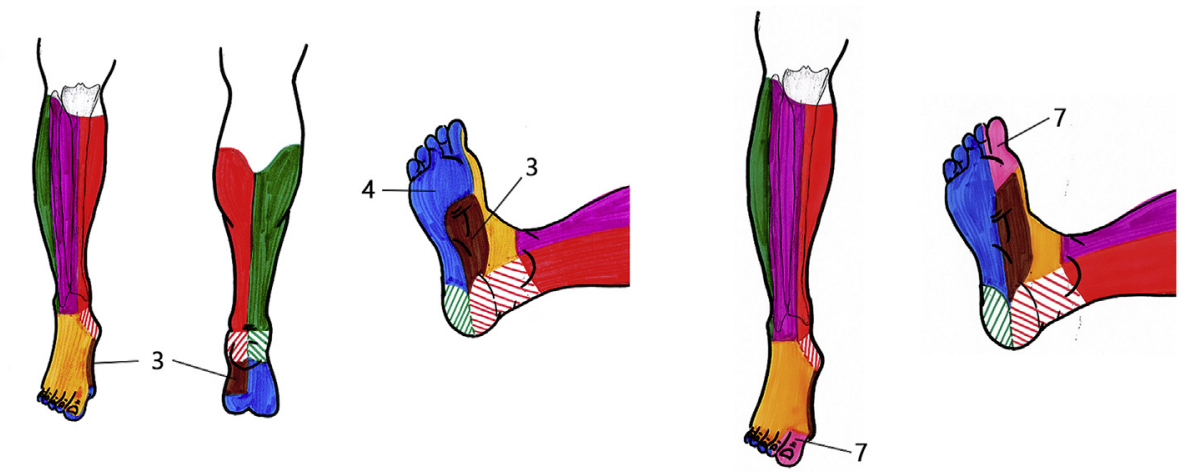

Fig. 1. (A) Angiosomal distribution used in present study. (B) Variations of angiosomal distribution. a: Anterior tibial artery and its angiosome; b: peroneal artery and its angiosome; and c: posterior tibial artery and its angiosome. 1: Dorsal angiosome, source a = ADP; 2 : dorsomedial and plantar angiosome, source $\mathrm{a}=$ calcanear branch of ATP; 3: medial plantar instep and forefoot angiosome, source $\mathrm{a}=$ medial plantar artery; 4 : lateral plantar foot

In 2006 Attinger et al. $^{2}$ studied the angiosome distribution in foot and concluded that foot contains 6 angiosome regions, each of which supplied by 1 of the 3 main crural arteries and their branches (Fig. 1). This knowledge has brought a new view on revascularization in patients with critical limb ischemia and so called angiosometargeted revascularization, meaning selective revascularization of the specific artery feeding the angiosome affected by ulcer, has been studied. ${ }^{3-7}$ Several retrospective studies comparing angiosome-targeted and angiosome-nontargeted approach in endovascular revascularization have been carried out, showing promising results in regard to wound healing and limb salvage in favor of the angiosome-targeted approach, especially in the diabetic foot. ${ }^{3-5,8-11}$

The purpose of this retrospective study was to evaluate the feasibility of the angiosome theory. We aimed to answer the questions of (1) how often the wound is located in more than just 1 angiosome, and forefoot angiosome, source $\mathrm{a}=$ lateral plantar artery; 5: dorsolateral and plantar angiosome, source $\mathrm{a}=$ calcanear branch of fibular artery; 6: lateral malleolar angiosome, source $\mathrm{a}=$ anterior perforating artery (part of the fibular artery angiosome); and 7: the hallux and medial side of the second toe angiosome, source artery $=$ dorsal metatarsal artery $(78 \%)$, plantar metatarsal artery $(22 \%)$.

and (2) how often angiosome-targeted endovascular revascularization is possible.

\section{MATERIAL AND METHODS}

This study was a retrospective analysis of prospectively collected data from a maintained vascular database (HUSVasc). It included all patients with CLI, classification Rutherford 5-6 (ischemic ulcer or gangrene and dry or humid), who underwent PTA of the arteries BTK during 2012. The angiograms of 182 consecutive endovascular procedures for CLI and tissue lesion were carefully analyzed before and after PTA and comparison was made between the anatomy of PTA and the wound location. Overall, 22 procedures were excluded because they either were re-PTA procedures to the same leg during the same year or the wound location was not clear. In the end, 161 procedures in 160 patients were included. One patient underwent procedure to the both legs. 
The wound locations were determined using the clinical notes and photographs stored in our database. As a result of an unclear distribution of the borderlines between angiosomes, due to the existence of more than one scheme, ${ }^{2,3,5,12}$ we decided to adopt the general scheme of angiosomal distribution, ${ }^{5}$ in which borderlines between angiosomes match those outlined in the recent publication; angiosomes application in critical limb ischemia in search for relevance by Alexandrescu ${ }^{13}$ (Fig. 1A).

We examined the number of crural arteries that were feasible for endovascular or surgical revascularization according to each patient's angiogram. The degree of disease in the crural vessels was described by using the Society for Vascular Surgery (SVS)/International Society for Cardiovascular Surgery (ISCVS) scoring system of angiographic appearance as follows: 0 , normal or minimal evidence of disease; 1, 20-49\% stenosis; 2, 50-99\% stenosis; 2.5 , occluded less than halfway, 3 , occluded through most of the length. ${ }^{14}$ At our clinic, the indication for PTA is stenosis of at least $50 \%$ and patent outflow after the affection (stenosis and/or occlusion) in the diseased crural artery (Fig. 2A-C). When the crural artery fulfilled these criteria, it was considered a candidate for endovascular treatment.

In the case of long occlusion, the endovascular treatment is usually attempted but if failed surgical revascularization is done or patient is scheduled primarily to surgical revascularization. This is always the case if distal outflow vessel exists. These cases were counted as feasible for angiosome-targeted revascularization. In case there was no patent outflow after the occlusion (pedal arteries not patent), it was not considered a candidate for revascularization.

After analyzing the number of BTK arteries feasible for endovascular treatment, we divided the patients into 3 groups: group 1 (Gl) representing patients with only 1 crural artery suitable for revascularization, group 2 (G2) containing patients with 2 crural arteries fulfilling the conditions for angioplasty, and group 3 (G3) containing patients with all 3 crural arteries as an option for revascularization. In each group, we examined the number of cases in which the targeted revascularization was feasible and performed, using the definition of angiosome-targeted revascularization from the recent publication. ${ }^{5}$ According to this definition, the revascularization is targeted if one of the source arteries leading to the wound area is open after the procedure even if the wound spreads over more than one angiosome. Meaning
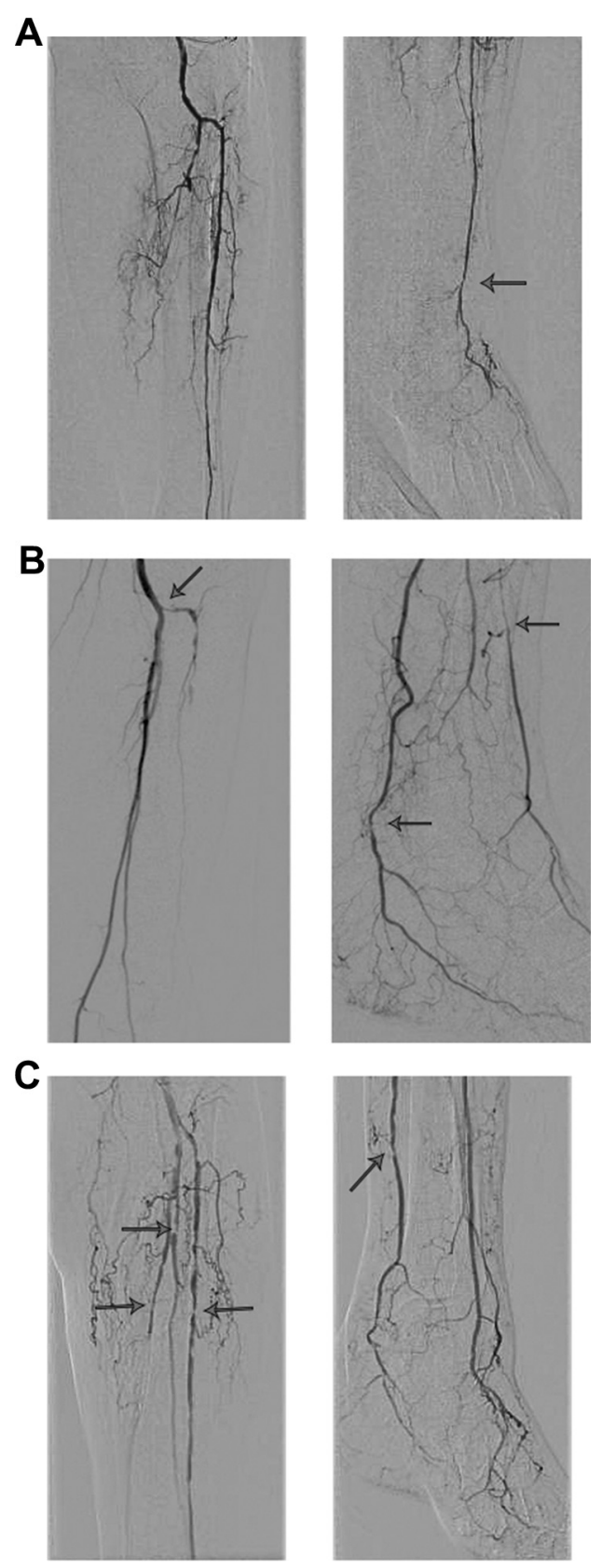

Fig. 2. (A-C) Angiograms showing the crural arteries suitable for PTA. (A) One crural artery suitable for PTA: $90 \%$ stenosis in distal anterior tibial artery (arrows); ATP and fibular artery completely occluded with no distal outflow. (B) Two crural arteries suitable for PTA: $67 \%$ stenosis in distal ATP and a 212-mm long occlusion of the ATA with present outflow distally (ADP fully patent), fibular artery without any sickness. (Arrows indicate the stenosis suitable for balloon dilatation.) (C) Three crural arteries suitable for PTA: pearllike ATA, 95\% stenosis in fibular artery and a 57.7$\mathrm{mm}$ long occlusion in the ATP with present distal outflow (arrows). 
if the ulceration is located in forefoot (tip of toes) angiosome-targeted revascularization is considered as either revascularization of arteria dorsalis pedis (ADP)/arteria tibialis anterior (ATA) or plantar artery/arteria tibialis posterior (ATP). If the ulceration spreads over plantar heel, angiosometargeted revascularization is considered if inflow to either calcanear branch rising from ATP or calcanear branch rising from fibular artery has been successfully achieved. This method does not pay attention to which of the arteries was the major source for affected area., ${ }^{5,15}$

In addition, the feasibility of angiosome-targeted revascularization in relation to number of angiosomes affected by ulcer, technical success rate, and the reasons why targeted revascularization was not achieved were analyzed.

Study was accepted in the ethical committee of Helsinki University Hospital.

\section{RESULTS}

A total of 161 legs (160 patients) undergoing primary infrapopliteal angioplasty were included. The mean age of the patients was 75.8 (42-93) and $37.5 \%$ were women. Basic demography of the patients is presented in the Table I. In majority ( $n=124 ; 77 \%)$ of the legs, the wound was classified as Rutherford 5 (Table I).

The wound(s) interfered with 1 angiosome in 39 $(24.2 \%)$ legs, with 2 angiosomes in $75(46.6 \%)$ legs, with 3 angiosomes in $42(26.1 \%)$ legs, with 4 angiosomes in $4(2.5 \%)$ legs, and with 5 angiosomes in 1 $(0.6 \%)$ legs Figure 3.

Of all extremities, 53 (32.9\%) had only l crural artery suitable for revascularization (Gl); the remaining 2 were either without any sign of disease $(n=17)$ or occluded $(n=36)$ with no patent outflow.

Two crural arteries (G2) fulfilling the criteria for revascularization were observed in 88 (54.7\%) legs. In only $20(12.4 \%)$ legs all 3 crural vessels were suitable for endovascular revascularization (G3) Table II.

The angiosome-targeted revascularization was possible in $129(80.1 \%)$ legs and not possible in 32 $(19.9 \%)$. Of the 129 patients in whom angiosometargeted revascularization was possible, it was performed and direct flow achieved in $98(75.9 \%)$ cases. The reasons for not performing angiosometargeted PTA when it was possible (31 cases) were as follows: (1) it was attempted without success $(n=9)$ and another vessel was then revascularized; (2) the occlusion was long and should have required bypass $(n=14)$, resulting in another vessel being
Table I. Patients characteristics and comorbidities

\begin{tabular}{|c|c|c|}
\hline Patients characteristics & \multicolumn{2}{|c|}{ Mean value } \\
\hline Age, years & \multicolumn{2}{|c|}{$75.8(42-93)$} \\
\hline Glomerular filtration & \multicolumn{2}{|c|}{$50.3(7-<60)$} \\
\hline Kreatinin & \multicolumn{2}{|c|}{$125.2(30-668)$} \\
\hline \multirow[t]{2}{*}{ CRP } & \multicolumn{2}{|c|}{$50.5(3-263)$} \\
\hline & Number of cases & $\%$ \\
\hline Female sex & 60 & 37.5 \\
\hline Smoking & 20 & 12.5 \\
\hline Diabetes & 107 & 66.9 \\
\hline Dyslipidemia & 88 & 55.0 \\
\hline Chronic kidney disease & 43 & 26.9 \\
\hline $3 \mathrm{~A}$ & 30 & 18.8 \\
\hline $3 B$ & 21 & 13.1 \\
\hline 4 & 4 & 2.5 \\
\hline 5 & 10 & 6.3 \\
\hline Dialysis & 14 & 8.8 \\
\hline Kidney transplantation & 7 & 4.4 \\
\hline Hypertension & 98 & 61.3 \\
\hline Atrial fibrillation & 59 & 36.9 \\
\hline Coronary artery disease & 63 & 39.4 \\
\hline Hearth failure & 24 & 15.0 \\
\hline Stroke & 28 & 17.5 \\
\hline Pulmonary disease & 20 & 12.5 \\
\hline Rutherford 5 & 124 & 77.0 \\
\hline Rutherford 6 & 37 & 23.0 \\
\hline
\end{tabular}

CRP, C-reactive protein.

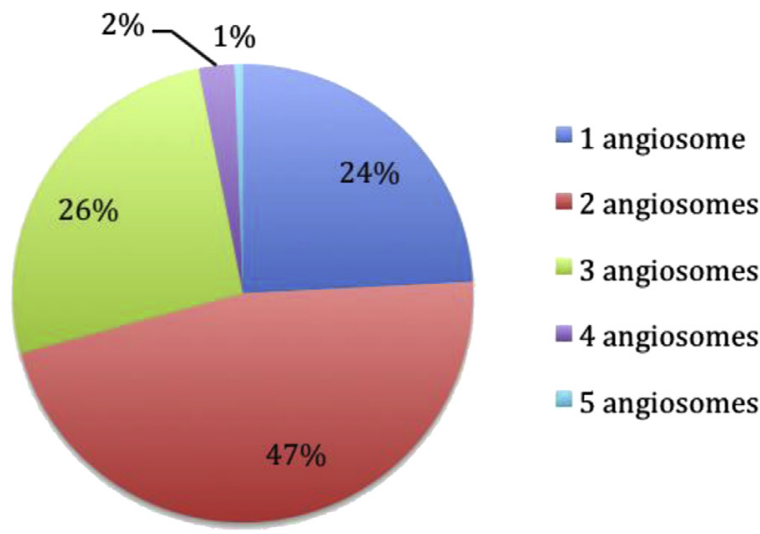

Fig. 3. The number of affected angiosomes in 161 legs.

revascularized; and (3) unknown reason $(n=8)$. Of all 161extremities, the direct flow was achieved in $98(60.9 \%)$ legs and indirect flow via nontargeted revascularization in $63(39.1 \%)$ legs Table II.

An analysis of the feasibility and technical success rate of targeted revascularization in relation to wound distribution showed that cases where wound spread over 2 or 3 angiosome had higher chance that the source artery will be feasible for 
Table II. Feasibility and success rate of targeted revascularization in relation to number of crural arteries suitable for PTA

\begin{tabular}{|c|c|c|c|c|c|c|}
\hline \multicolumn{7}{|l|}{ Method a } \\
\hline \multirow[b]{2}{*}{ Artery suitable for PTA } & \multirow{2}{*}{$\begin{array}{l}\text { Number } \\
\text { of cases }\end{array}$} & \multirow{2}{*}{$\begin{array}{l}\text { Targeted } \\
\text { possible }(\%)\end{array}$} & \multirow{2}{*}{$\begin{array}{l}\text { Targeted } \\
\text { achieved }(\%)\end{array}$} & \multicolumn{3}{|c|}{ Targeted not achieved } \\
\hline & & & & Failed (\%) & Long occlusion (\%) & Unknown $(\%)$ \\
\hline 1 Crural artery (Gl) & 53 & $33(62.3)$ & $26(78.8)$ & $2(6.1)$ & $3(9.1)$ & $2(6.1)$ \\
\hline 2 Crural arteries (G2) & 88 & $76(86.4)$ & $57(75.0)$ & $6(7.9)$ & $8(10.5)$ & $5(6.6)$ \\
\hline 3 Crural arteries (G3) & 20 & $20(100.0)$ & $15(75.0)$ & $1(5.0)$ & $3(15.0)$ & $1(5.0)$ \\
\hline Total & 161 & $129(80.1)$ & $98(75.9)$ & $9(7.0)$ & $14(10.9)$ & $8(6.2)$ \\
\hline
\end{tabular}

Table III. Feasibility and success rate of targeted revascularization in relation to wound distribution

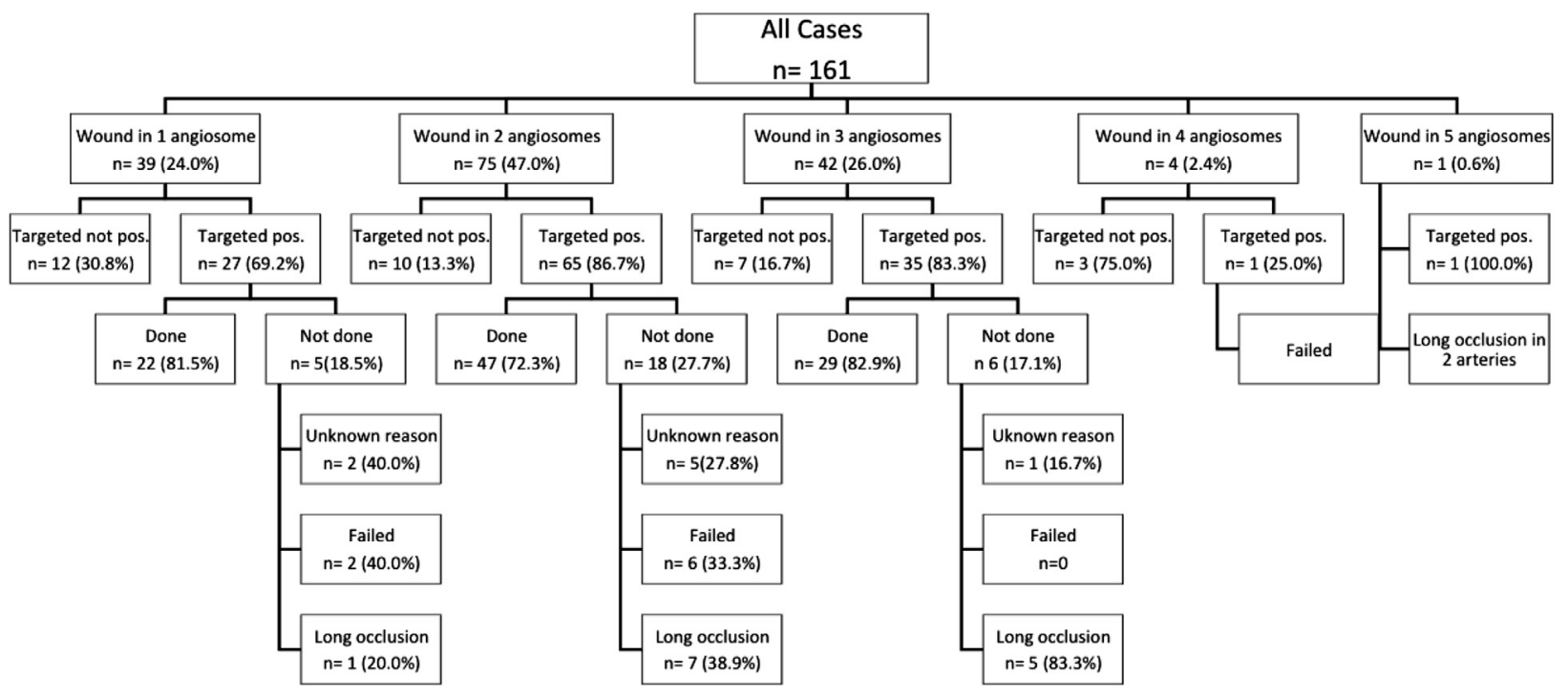

angiosome-targeted revascularization: if ulceration was limited in 1 angiosome, the revascularization was possible in 27 cases $(69.2 \%)$, if ulceration was extended to 2 angiosomes it was possible in 65 cases $(86.7 \%)$, if 3 angiosomes were affected it was possible in cases $36(85.7 \%)$, if ulceration was spread over 4 angiosomes it was possible in 1 of 4 cases, and ulceration spread over 5 angiosome $(n=1)$ had no possibility of revascularization, detailed presented in Table III.

\section{DISCUSSION}

The importance of angiosome-targeted revascularization has been lively discussed during recent years. Quite a few studies have found targeted revascularization an important factor associated with wound healing, but opposite opinions also exist. ${ }^{16}$ We studied the feasibility of angiosome-targeted revascularization in the endovascular treatment of critical limb ischemia and tissue lesions. Our findings show, that the wound(s) are not located only in one angiosome but that, more likely, they affect several angiosomes. Furthermore in every third patient, there was only one crural artery suitable for revascularization. However, in majority of the patients angiosome-targeted revascularization seems to be possible.

The reason for why 2 or more angiosomes are affected is most often the fact that the wound is located on the borderline of 2 or even more angiosomes or that the patient suffers from more than one ulcer in the foot. When examining previous studies, $4,5,10,17$ we lacked the definition of the angiosome-targeted approach in those cases where the wound spreads over more angiosome regions. In a study by Lida et al. targeted revascularization was defined as an open line from the abdominal aorta to the artery supplying the angiosome where the wound was located, with no mention of strategy for cases with ulcer affecting 2 or more angiosomes. ${ }^{4}$ 
The study done by Söderström et al. ${ }^{5}$ points out that the wound can be located across more than one angiosome, but do not provide clear definition of targeted revascularization in those cases.

In cases where wound spread over more angiosomes the conventional method used in most of the publications consider as angiosome-targeted approach if any angiosomal artery involved in the wound area is opened after procedure. ${ }^{5}$ That allows the clinician to choose from more than 1 crural artery, and thus, there is higher possibility to achieve targeted revascularization. Such an approach may be based on knowledge from a Japanese anatomic study, ${ }^{18}$ which demonstrated that in $78 \%$ of cases, the blood supply to the forefoot is received from the first dorsal metatarsal artery (rising from ADP) and only in $22 \%$ from the first plantar metatarsal artery (rising from lateral plantar artery).

Alexandrescu $^{13}$ recently presented another angiosome-targeted approach, which targets only posterior tibial artery and its terminal branches if the wound is located in forefoot or the heel. This would most probably lead to a lowered probability that angiosome artery will be a possible candidate for endovascular treatment as it results in only 1 crural artery being a choice. Such an approach may be based on knowledge that in $12 \%$ of population, the ADP is extremely thinned or absent. ${ }^{3,6,18}$

In the present study, although there was distal outflow to the angiosome that was affected by a wound, revascularization was not performed in $20.0 \%(n=31)$ of the cases. In about half of the cases, the approach was attempted without success and an easier artery was then selected; and in about one-third of cases it was not even attempted because of a long lesion, and a vessel providing easier revascularization was selected. In these cases, angiosometargeted revascularization could have been achieved with open surgical bypass. In 8 cases $(25.8 \%)$, there was no explanation as to why targeted revascularization was not preformed and another artery was revascularized instead, although the targeted revascularization seemed possible according to the angiograms recorded. The most probable explanation is a poor knowledge of the anatomy of wound location.

When the treatment policy is "endovascular first", it seems that we have to accept a certain number of nontargeted revascularizations, instead of treating suitable cases by targeted bypass.

The limitation of our study and the other studies on this topic is related to the angiosomal distribution. Because of multiple anatomic variations in the foot arteries, ${ }^{3,6}$ the general scheme of angiosomal distribution cannot be taken for granted in the case of every patient. The diversity of the distribution is well demonstrated in Attinger's study from 2006 where he found and described 2 major variations in the perfusion of the plantar heel and plantar forefoot. ${ }^{2}$ Furthermore, some of the authors have even considered the hallux and middle side of the second toe as a separate angiosome ${ }^{3,12}$ (Fig. 1). Therefore, a patient's anatomy should be examined carefully and considered in the decision of which artery should be revascularized. This may become problematic, if not impossible, in patients with a so-called desert foot (no patency of pedal arteries on angiogram).

Although we think that the angiosome concept is very logical and has considerable a lot of potential, we still face uncertainties in definitions and angiosome borders distribution when trying to apply it to treatment of CLI.

This study was retrospective, and the wound locations were extracted from case records. Although wound location has been carefully reported in our institution ever since the emergence of the interest in angiosomes and wound location appeared in the literature, the determination of the affected angiosome can be difficult in some cases. Photographs were included in the case histories of many of the patients included in the present study, which was very helpful. Moreover, in some cases, the evaluation of angiograms may be problematic because of the poor quality of the images as regards limitations in the examined region.

A well planned prospective study where the wound location is defined precisely, high quality angiogram with the information on collaterals and the patency of pedal arch is definitely needed.

\section{CONCLUSIONS}

In critical limb ischemia, the tissue lesion affects several angiosomes in majority. There is existence of more angiosome-targeted approaches, and therefore, consensus needs to be achieved for the accurate definition of angiosome-targeted revascularization when more than one angiosome is clinically involved.

\section{REFERENCES}

1. Taylor GI, Palmer JH. The vascular territories (angiosomes) of the body: experimental study and clinical applications. Br J Plast Surg 1987;40:113-41.

2. Attinger CE, Evans KK, Cooper P. Angiosomes of the foot and ankle and clinical implications for limb salvage: reconstruction, incisions, and revascularization. Plast Reconstr Surg 2006;117(Suppl):261S-93S. 
3. Houlind $\mathrm{K}$, Christensen J. The role of the angiosome model in treatment of critical limb ischemia, Artery Bypass, Dr Wilbert S. Aronow (Ed.), 2013;ISBN: 978-953-51-1025, InTech.

4. Iida $\mathrm{O}$, Nanto $\mathrm{S}$, Uematsu M. Importance of the angiosome concept for endovascular therapy in patients with critical limb ischemia. Cathet Cardiovasc Intervent 2010;75:830-6.

5. Söderström M, Albäck A, Biancari F, et al. Angiosome-targeted infrapopliteal endovascular revascularization for treatment of diabetic foot ulcers. J Vasc Surg 2013;57:427-35.

6. Bosanquet DC, Glasbey JC, Williams IM, Twine CP. Systematic review and meta-analysis of direct versus indirect angiosomal revascularisation of infrapopliteal arteries. Eur J Vasc Endovasc Surg 2014;48:88-97.

7. Biancari F, Juvonen T. Angiosome-targeted lower limb revascularization for ischemic foot wounds: systematic review and meta-analysis. Eur J Vasc Endovasc Surg 2014;47:517-22.

8. Alexandrescu V, Hubermont G. Primary infragenicular angioplasty for diabetic neuroischemic foot ulcers following the angiosome distribution: a new paradigm for the vascular interventionist? Diabetes Metab Syndr Obes 201 1;4:327-36.

9. Alexandrescu VA, Hubermont G, Horion J. Selective primary angioplasty following an angiosome model of reperfusion in the treatment of Wagner 1-4 diabetic foot lesions: practice in a multidisciplinary diabetic limb service. J Endovasc Ther 2008;15:580-93.

10. Serra R, Grande R, de Franciscis S. Angiosome-targeted revascularization in diabetic foot ulcers. Int Wound $\mathrm{J}$ $2015 ; 12: 555-8$.
11. Iida $O$, Takahara $M$, Uematsu M. Worse limb prognosis for indirect versus direct endovascular revascularization only in patients with critical limb ischemia complicated with wound infection and diabetes mellitus. Eur J Vasc Endovasc Surg 2013;46:575-82.

12. Manzi M, Cester G, Ferraresi R. First Step toward Endovascular Recanalization in Vascular Imaging of the Foot: http:// radiographics.rsna.org/lookup/suppl/doi:10.1148/rg.316115 $511 /-/ D C 1$.

13. Alexandrescu V. Angiosomes application in critical limb ischemia in search for relevance. Turin: Edizioni Minerva MedicaS.p.A, 2013. pp 1-11.

14. Rutherford R, Baker J, Ernst C, et al. Recommended standards for reports dealing with lower extremity ischemia; revised version. J Vasc Surg 1997;26:517-38.

15. Kret MR, Cheng D, Landry GJ. Utility of direct angiosome revascularization and runoff scores in predicting outcomes in patients undergoing revascularization for critical limb ischemia. J Vasc Surg 2014;59:121-8.

16. Varela C, Acín F, March JR. The role of foot collateral vessels on ulcer healing and limb salvage after successful endovascular and surgical distal procedures according to an angiosome model. Vasc Endovasc Surg 2010;44: 654-60.

17. Alexandrescu V, Söderström M, Venermo M. Angiosome theory: fact or fiction? Scand J Surg 2012;101:125-31.

18. Adachi B, Hasebe K. Das Arteriensystem der Japaner. Tokyo: Verl. der Keiserlich-Japanischen Universität zu Tokyo, 1928. 